\section{Beneficios de la suplementación con L-carnitina en pacientes pediátricos hemodializados. Reporte de casos}

Martínez-Morales JA ${ }^{1}$, Raya-Jiménez $\mathrm{P}^{2}$, Monroy-Torres $\mathrm{R}^{1}$, Daza-Benítez $\mathrm{L}^{2}$

\begin{abstract}
Resumen
INTRODUCCIÓN: la mayoría de los pacientes pediátricos con insuficiente renal crónica desarrollan desnutrición y anemia. Para tratar esta última se administra eritropoyetina como tratamiento de elección, pero no se logra recuperar la situación nutricional del paciente, por lo que la L-carnitina es una alternativa que mejora el estado nutricional $y$, al mismo tiempo, disminuye la frecuencia de anemia.
\end{abstract}

OBJETIVO: mejorar los parámetros bioquímicos y antropométricos después de la suplementación con L-carnitina en pacientes pediátricos.

MATERIAL Y MÉTODOS: se realizó un estudio analítico y longitudinal, de una serie de casos en 25 participantes, ambos sexos, de 3 a 16 años de edad, con insuficiencia renal crónica y en tratamiento de hemodiálisis. Se les suministró L-carnitina por vía intravenosa $(50 \mathrm{mg} /$ kg/sesión) 30 minutos antes de terminar las sesiones de hemodiálisis, durante tres meses (36 sesiones). Antes y después de la maniobra se compararon algunos indicadores bioquímicos (hemoglobina, albumina, colesterol, triglicéridos, creatinina) y antropométricos. Se realizó una comparación con T de Student y por proporciones (casos que mejoraron después de maniobra y la reducción del riesgo absoluto).

RESULTADOS: la edad promedio de los casos fue 12.4 años (rango de 3 a 16 años). Al comparar los casos con T de Student se obtuvo una mejora significativa con la suplementación para el peso seco $(p=0.004)$; índice de masa corporal $(p=0.04)$; albúmina $(p=0.02)$ y colesterol $(p=0.003)$. Con el cálculo por proporciones de los casos que mejoraron con la maniobra se observó la reducción del riesgo atribuible en un rango de 12 a $68 \%$ de todas las variables de interés (hemoglobina, albúmina, colesterol, triglicéridos, peso seco e índice de masa corporal).

CONCLUSIONES: la suplementación con L-carnitina intravenosa (50 mg/kg/sesión) 30 minutos antes de terminar cada sesión de hemodiálisis, durante tres meses, permitió una mejora en el porcentaje de los casos de estudio para peso seco, índice de masa corporal, albúmina, hemoglobina, colesterol y triglicéridos, con una reducción del riesgo atribuible de 12 a $68 \%$ de los casos.

PALABRAS CLAVE: insuficiencia renal crónica, anemia, suplementación, L-carnitina.

\footnotetext{
${ }^{1}$ Universidad de Guanajuato, Campus León. ¿Unidad Médica de Alta Especialidad, UMAE Hospital Gineco/pediatría No.48, León, Guanajuato.
}

Recibido: 15 de mayo del 2015

Aceptado: 28 de abril del 2016

\section{Correspondencia}

LN Jacqueline Alejandra Martínez Morales jacky_alex_109@hotmail.com

Este artículo debe citarse como Martínez-Morales JA, Raya-Jiménez P, MonroyTorres R, Daza-Benítez L. Beneficios de la suplementación con L-carnitina en pacientes pediátricos hemodializados. Reporte de casos. Acta Pediatr Mex. 2016;37(5):260-270. 


\section{Benefits of L-carnitine supplementation in pediatric hemodialysis patients: Cases report.}

Martínez-Morales JA ${ }^{1}$, Raya-Jiménez $\mathrm{P}^{2}$, Monroy-Torres $\mathrm{R}^{1}$, Daza-Benítez $\mathrm{L}^{2}$

\begin{abstract}
INTRODUCTION: Most pediatric patients with chronic renal failure develop mal nutrition and anemia. To treat anemia, erythropoietin (EPO) is administered as a treatment of choice, but does not recover the nutritional status of the patient, so that L-carnitine, is presented as an alternative that improves the nutritional status, while It decreases the frequency of anemia.
\end{abstract}

OBJECTIVE: To improve the biochemical and anthropometric parameters, after supplementation with L-carnitine in pediatric patients.

MATERIAL AND METHODS: A longitudinal study, case series in 25 participants, both sexes, aged 3-16 years old, with chronic renal failure and hemodialysis was performed. They were provided L-carnitine i.v. (50 mg / kg / session), 30 minutes before finishing hemodialysis sessions for three months (36 sessions). Before and after the maneuver some biochemical parameters (hemoglobin, albumin, cholesterol, triglycerides, creatinine) and anthropometric were compared. a comparison with T Student and proportions (cases improved after switching and absolute risk reduction) was performed mainly.

RESULTS: The average age of cases was 12.4 years (range 3 -16 years). When comparing cases with T student, a significant improvement with supplementation for dry weight $(p=0.004)$, body mass index $(p=$ $0.04)$, albumin $(p=0.02)$ and cholesterol $(p=0.003)$ was obtained. With the calculation proportions of cases improved with the maneuver, reducing the risk attributable observed in a range from 12 to $68 \%$ of all of the variables of interest (hemoglobin, albumin, cholesterol, triglycerides, dry weight and $\mathrm{BMI}$ ).

CONCLUSIONS: Supplementation connote-carnitine I.V. 50 mg / kg / session, 30 minutes before the end of each hemodialysis session for three months, allowed an improvement in the percentage of case studies for dry weight, BMI, albumin, hemoglobin, cholesterol and triglycerides, a reduction attributable risk of 12 to $68 \%$ of cases.

KEYWORDS: chronic renal failure; anemia; supplementation; Lcarnitine

\footnotetext{
${ }^{1}$ Universidad de Guanajuato, Campus León. ${ }^{2}$ Unidad Médica de Alta Especialidad, UMAE Hospital Gineco/pediatría No.48, León, Guanajuato.
}

\section{Correspondence}

LN Jacqueline Alejandra Martínez Morales jacky_alex_109@hotmail.com 


\section{INTRODUCCIÓN}

En América Latina, en el 2010, la incidencia de enfermedad renal fue de 2.6 a 15.8 casos por cada millón de habitantes en menores de 15 años. En el 2012, en México, la incidencia fue de 3.5 por cada millón de habitantes menores de 15 años ${ }^{1}$ y cada año se diagnostican 100 mil casos de insuficiencia renal, donde 12 mil de los detectados están en etapa terminal y sólo un $30 \%$ son candidatos a trasplante renal. ${ }^{1}$

Una vez que el paciente inicia una terapia de sustitución (diálisis o hemodiálisis) se inicia también un deterioro nutricio con una pérdida de nutrimentos, principalmente aminoácidos, albúmina, vitaminas hidrosolubles y carnitina. ${ }^{2}$ El desarrollo de anemia normocítica y normocrómica es una complicación en los pacientes con insuficiencia renal crónica, ${ }^{3}$ que se trata con eritropoyetina humana recombinante; ${ }^{4}$ sin embargo, muchos pacientes siguen presentando baja respuesta a pesar del tratamiento adecuado con hierro. ${ }^{3,4}$ Varios estudios han demostrado que la suplementación con carnitina en pacientes con insuficiencia renal crónica evita las complicaciones cardiológicas, mejora la sintomatología muscular, la hipotensión intradialítica y la frecuencia de anemia que no mejora con la eritropoyetina humana recombinante. ${ }^{5} \mathrm{En}$ 1999, la Food and Drug Administration aprobó el empleo de L-carnitina en pacientes con desordenes dialíticos y para mejorar la respuesta a la administración con eritropoyetina humana recombinante. ${ }^{6}$ En 2011 México la autorizó ante la Comisión Federal para la Protección contra Riesgos Sanitarios. ${ }^{7}$

La carnitina es una amina cuaternaria, soluble en agua, no unida a albúmina, la isoforma levo es biológicamente activa, mejor conocida como Lcarnitina, cuya importancia radica en su función principal en el metabolismo de los ácidos grasos y en la producción de energía. ${ }^{8}$ El mecanismo es que la L-carnitina transporta los ácidos grasos que se encuentra en el citosol de la célula, para que puedan entrar a la matriz mitocondrial y así comenzar el proceso de $\beta$ oxidación de los ácidos grasos y obtener acetilcoenzima (CoA) y producir energía a través del ciclo de krebs. ${ }^{9}$ La principal función de la L-carnitina es la producción de energía, principalmente en riñón, hígado, cerebro y músculo esquelético, así como la de actuar como antioxidante, al reducir la peroxidación lipídica y, por ende, reduce el daño potencial a la membrana celular durante el estrés oxidativo. ${ }^{8}$

La L-carnitina se filtra libremente por la membrana glomerular y se reabsorbe por el túbulo proximal a través de un catión orgánico, con un aclaramiento renal de 1-3 mL/min. En contraste, durante la hemodiálisis $(100 \mathrm{~mL} / \mathrm{min})$, la L-carnitina se disminuye en el plasma aproximadamente en un $80 \%$ al final de cada sesión. ${ }^{10}$ Esta reducción en el plasma se debe a que la síntesis endógena se ve alterada durante la hemodiálisis, así como a la deficiencia de algunos cofactores (vitamina B6, niacina, vitamina $\mathrm{C}$ y hierro) que son necesarios en la ruta enzimática encargada de sintetizar carnitina. ${ }^{10}$

La L-carnitina podrían influir en la respuesta a la eritropoyetina humana recombinante, al participar en los procesos de desacilación y reacilación que permiten remodelar membranas de fosfolípidos en los eritrocitos, por ende, la estimulación de la eritropoyesis en altas concentraciones ( $>200 \mathrm{mmol} / \mathrm{L}$ ) genera un incremento en la sobrevida de los eritrocitos al reducir el estrés oxidativo. ${ }^{11}$

Hurot JM y sus colaboradores, en una revisión sistemática sobre la utilidad de la L-carnitina en pacientes con insuficiencia renal crónica con hemodiálisis, no encontraron un beneficio con la suplementación de L-carnitina sobre los niveles de triglicéridos y colesterol, pero sí observaron 
una mejora en los niveles de hemoglobina y una menor dosis de eritropoyetina humana recombinante. $^{8}$

Otros estudios demostraron que la suplementación con L-carnitina en adultos, durante seis meses, mejoró la osmosis de la membrana celular de los eritrocitos. ${ }^{9}$ Se conoce que la L-carnitina tiene una función importante en el tratamiento de pacientes con insuficiencia renal crónica, para disminuir la anemia, ya que la hemólisis ocurre exclusivamente en pacientes con bajos niveles séricos de carnitina. ${ }^{10}$ En el estudio realizado por Aoun y su grupo a seis pacientes pediátricos con hemodiálisis, les fue administrada L-carnitina intravenosa (2.5 g por sesión para los pacientes de más de $30 \mathrm{~kg}$ y un $1 \mathrm{~g}$ para los que presentaron un peso menor a $30 \mathrm{~kg}$ ) durante 9 meses. Al finalizar los estudios los niveles de hemoglobina aumentaron y disminuyeron los requerimientos de eritropoyetina humana recombinante, sin efectos secundarios. ${ }^{11}$

\section{OBJETIVO}

Por la evidencia ya mencionada y a la necesidad de contar con esquemas y opciones de tratamiento que mejoren y optimicen el estado nutricio y el pronóstico de la insuficiencia renal crónica en el paciente pediátrico, se realizó este estudio en un hospital público de León, Guanajuato, donde se buscó probar la hipótesis de que la suplementación con L-carnitina en pacientes pediátricos hemodializados lograba una mejoría en los marcadores bioquímicos (hemoglobina, albúmina, colesterol, triglicéridos, creatinina) y antropométricos (peso seco e índice de masa corporal).

\section{MATERIAL Y MÉTODOS}

Se realizó un estudio de reporte de casos, analítico y longitudinal, durante tres meses, en el área de hemodiálisis pediátrica de la unidad médica de alta especialidad de Gineco/Pediatra del IMSS, número 48, de la ciudad de León, Gto. Se incluyeron a 25 pacientes (casos) por simple disponibilidad, cuyos criterios de inclusión fueron: pacientes pediátricos entre 3 y 16 años de edad, ambos sexos, con diagnóstico de insuficiencia renal crónica, en tratamiento con hemodiálisis. No se incluyeron a pacientes con sepsis, candidatos a trasplante o que estuvieran recibiendo L-carnitina al momento o dos meses previos al estudio. Se excluyeron a los participantes que fallecieran o que presentaran en el transcurso alguna complicación grave relacionada a su padecimiento, así como el desarrollo de sepsis y hemorragias.

Una vez que los padres aceptaron y firmaron el consentimiento informado de sus hijos, se procedió a evaluar el estado nutricio, con lo que se obtuvieron los indicadores antropométricos, al inicio y a los tres meses, de la suplementación con L-carnitina; las mediciones se realizaban al término de las sesiones de hemodiálisis. Se obtuvo el peso seco, utilizando una báscula de marca Torino ${ }^{\circledR}$, con precisión de 100 g. Para la estatura se utilizó un infantómetro marca Seca ${ }^{\circledR}$, precisión $0.1 \mathrm{~cm}$, para los niños que tuvieran menos de un metro; mientras que para los mayores de un metro la medición se realizó por medio de un estadímetro de la marca $\operatorname{Seca}^{\circledR}$, con una precisión $0.1 \mathrm{~cm} .{ }^{12}$ Con el peso y talla se obtuvo el índice de masa corporal y los valores se interpretaron con las tablas de percentiles para niñas y niños, así como las de referencia para adolescentes de 2 a 20 años, de los Centers for Disease Control and Prevention. Para el pliegue cutáneo tricipital (PCT) la medición se realizó por duplicado utilizando el plicómetro Lange ${ }^{\circledR}$, con una precisión de $1 \mathrm{~mm}$. Se midió la circunferencia del brazo (CB) con una cinta métrica de fibra de vidrio, marca Seca $201^{\circledR}$, precisión $0.5 \mathrm{~mm}$ (25). Con el pliegue cutáneo tricipital y la circunferencia del brazo se obtuvo el área muscular de brazo (AMB) con la siguiente fór- 
mula: $\mathrm{AMB}=\left[\mathrm{PB}-\left(\pi^{*} \mathrm{PCT}\right)\right]^{2} / 4 \pi$. Posteriormente, se calculó el área adiposa del brazo $(A A B)$ con la siguiente fórmula: $\mathrm{AAB}=\mathrm{AMB}-\mathrm{ATB}$; para obtener el área total del brazo (ATB) se utilizó (CB mm/6.2832) $2 * \pi$. Los resultados se analizaron en base a la tabla de percentiles del área muscular de brazo para edad y sexo $\left(\mathrm{mm}^{2}\right)$ de los Centers for Disease Control and Prevention. Las técnicas de medición fueron acorde las

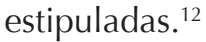

Respecto a la suplementación con L-carnitina, se aplicó Cardispan I.V., que es L-carnitina 50 mg/ $\mathrm{kg} /$ sesión y se preparó en un mililitro de solución salina, en una jeringa de $20 \mathrm{~mL}, 30$ minutos antes de terminar cada sesión de hemodiálisis y se aplicó sobre la línea venosa. ${ }^{11}$ El cardispan fue proporcionado por la Unidad Médica de Alta Especialidad, UMAE Hospital Gineco/pediatría 48 (UMAE 48) y fue aplicado por las enfermeras del área de hemodiálisis pediátrica.

Los indicadores bioquímicos se obtuvieron del expediente médico, donde se registraron los niveles de hemoglobina durante tres meses del estudio, así como la cantidad de eritropoyetina administrada y los indicadores metabólicos (bioquímicos) como la biometría hemática para la medición de hemoglobina, albúmina sérica, colesterol, triglicéridos, sodio, potasio, calcio, fósforo, urea y creatinina; todos se registraron al inicio y al final del estudio (tres meses). Los análisis fueron realizados por personal capacitado del laboratorio de análisis clínicos de la UMAE 48.

\section{Análisis estadístico y aspectos de ética}

Para el análisis estadístico se utilizó el programa estadístico SPSS $15^{\circledR}$ (2012). Se aplicó estadística descriptiva para las variables antropométricas y bioquímicas, excepto para la interpretación del índice de masa corporal; la distribución por sexo se hizo por porcentajes. Para la comparación de los indicadores antropométricos y bioquímicos pre y post de la aplicación con L-carnitina IV se utilizó una prueba de t de student, así como una correlación de Pearson para las variables antropométricas y bioquímicas. Se procedió a una prueba de Rangos de Wilcoxon, para la comparación de la eritropoyetina y el paquete globular, pre y post la suplementación con Lcarnitina. Finalmente, se realizó un cálculo de la reducción del riesgo atribuible (siendo cada caso el mismo control, pre y post de la maniobra con L-carnitina) y una comparación por proporciones (con el paquete estadístico ${ }^{\circledR}$ PRIMER McGrawHill Interamericana, 1998) para: peso seco, índice de masa corporal, hemoglobina, albúmina, colesterol y triglicéridos. Se consideró mejoría cuando hubo un incremento en los valores de las variables de relevancia, a los tres meses de la suplementación: para peso seco un incremento con un valor $\geq$ a $0.5 \mathrm{~kg}$ ); para la hemoglobina $\geq$ a $1 \mathrm{~g} / \mathrm{dL}$; para el índice de masa corporal y albúmina que modificaran los valores; para colesterol y triglicéridos que se mostrara una reducción de las cifras iniciales, $\mathrm{p}<0.05$ y un $\beta$ del $80 \%$. El proyecto fue aprobado por el Comité de Investigación y Ética del IMSS (Folio local: F-2012-1002-58 y Folio nacional: R-2012-1002-55). Los procedimientos de esta investigación fueron de acuerdo a los lineamientos estipulados por la ley general de la salud en materia de investigación en seres humanos, donde se aplicó el consentimiento informado. La L-carnitina ha sido utilizada previamente en este tipo de pacientes, por lo que se cuidó no poner en riesgo a los participantes.

\section{RESULTADOS}

De los 25 casos estudiados con insuficiencia renal crónica en hemodiálisis 11 fueron del sexo femenino y 14 del sexo masculino, con un promedio de edad de 12.4 años (3 a 16 años) (Cuadro 1). Al realizar la comparación con los indicadores antropométricos pre y post de la aplicación con L-carnitina IV (T de Student), 
Cuadro 1. Características basales de los participantes

\begin{tabular}{l|c} 
Variable & $\begin{array}{c}\text { Pre aplicación L- carnitina } \\
\text { Media }\end{array}$ \\
\hline Edad & 12 años 2 meses \\
\hline Sexo & $14(56)$ \\
H n $\%)$ & $11(44)$ \\
M n $(\%)$ & $29.148 \pm 12.84 \mathrm{~kg}$ \\
\hline Peso seco & $1.27 \pm .17 \mathrm{~m}$ \\
\hline Talla & $19.24 \pm 4.51 \mathrm{~cm}$ \\
\hline CB & $6.58 \pm 4.11 \mathrm{~mm}$ \\
\hline PCT & $16.89 \pm 3.85 \mathrm{~kg} / \mathrm{m}^{2}$ \\
\hline IMC & $11(44)$ \\
\hline$<$ Percentil $25 \mathrm{n}(\%)$ & $9(36)$ \\
$\geq$ percentil $50 \mathrm{n}(\%)$ & $5(20)$ \\
Percentil $25 \mathrm{n}(\%)$ & $2401.60 \pm 1033.59 \mathrm{~mm}{ }^{2}$ \\
\hline AMB & $675.57 \pm 530.29 \mathrm{~mm}{ }^{2}$ \\
\hline AAB & $8.40 \pm 1.55 \mathrm{~g} / \mathrm{L}$ \\
\hline HB & $4.00 \pm .59 \mathrm{~L} / \mathrm{L}$ \\
\hline HTO & $26.56 \pm 4.73 \mathrm{mg} / \mathrm{L}$ \\
\hline Albúmina & $151.28 \pm 44.95 \mu \mathrm{mol} / \mathrm{L}$ \\
\hline TGC & $148.24 \pm 41.94 \mu \mathrm{mol} / \mathrm{L}$ \\
\hline Colesterol & $140.56( \pm 4.60) \mathrm{mEq} / \mathrm{L}$ \\
\hline Na & $4.86 \pm 1.12 \mathrm{mEq} / \mathrm{L}$ \\
\hline K & $8.98 \pm 1.28 \mathrm{mg} / \mathrm{dL}$ \\
\hline CA & $5.44 \pm 1.89 \mathrm{mg} / \mathrm{dL}$ \\
\hline P & $121.21 \pm 40.44 \mathrm{mg} / \mathrm{dL}$ \\
\hline Urea & $7.81 \pm 2.43 \mathrm{mg} / \mathrm{dL}$ \\
\hline creatinina & \\
\hline
\end{tabular}

CB: pliegue cutáneo tricipital; IMC: índice de masa corporal; AMB: área muscular de brazo; AAB: área adiposa de brazo; HB: hemoglobina; HTO: hematocrito; TGC: triglicéridos; Na: sodio; K: potasio; Ca: calcio; P: fósforo.

se encontró una diferencia significativa para peso seco e índice de masa corporal $(p=0.04)$ (Cuadro 2). Respecto a la comparación para los indicadores bioquímicos se observó una diferencia significativa para todos los marcadores bioquímicos (albúmina, colesterol, triglicéridos, fósforo y creatinina) excepto para la hemoglobina. Para el caso de la albúmina esta diferencia detectada se debió porque al final disminuyó sus valores; para el caso del colesterol y triglicéridos esta diferencia fue por un incremento al final de la maniobra (Cuadro 3).

Respecto a la comparación con las dosis del paquete globular y la aplicación de eritropoyetina humana recombinante pre y postmaniobra, sólo se observó una diferencia significativa para el paquete globular $(p=0.003)$ (Cuadro 4).

Respecto al análisis de correlación hubo una relación positiva para las variables antropométricas de peso seco, talla y área muscular de brazo con la creatinina final; el resto de las variables se muestra en el Cuadro 5.

$\mathrm{Al}$ analizar el porcentaje de casos que se vieron beneficiados con la mejora en los indicadores antropométricos y bioquímicos, después de la suplementación con L-carnitina y que fueran de interés clínico de acuerdo a los expuesto en la evidencia científica, se pudo observar un cambió para la hemoglobina, el peso seco, albúmina de los casos que mejoraron sus cifras después de la maniobra; para el caso de la albúmina se observó un incremento en 17 de los casos (68\%), se observó un incremento de las cifras de albúmina de 0.1 a $2 \mathrm{~g} / \mathrm{dL}$, y se mantuvo el valor dentro de lo adecuado en el $96 \%$ de los casos (Cuadro 5). Las cifras de hemoglobina en 15 de los casos presentaron un incremento al final de la maniobra de 0.1 a $2.9 \mathrm{~g} / \mathrm{dL}$, pero sólo en diez casos se observó un incremento de $1 \mathrm{~g} / \mathrm{dL}$.

Para los triglicéridos, en siete casos se observó una disminución después de la maniobra de 15 a $81 \mathrm{mg} / \mathrm{dL}$. Para los valores de colesterol, en 15 $(60 \%)$ de los casos se detectó una reducción de los valores de 6 a $54 \mathrm{mg} / \mathrm{dL}$, pero los casos que se mantuvieron con reducciones acorde a las recomendaciones se presentan en el Cuadro 6, donde la reducción en el riesgo en cualquiera de los marcadores antropométricos y bioquímicos mencionados fue del 12 al 68\% (Cuadro 6). 
Cuadro 2. Comparación en los indicadores antropométricos pre y post de la aplicación con L-carnitina IV en los participantes $(n=25)$

\begin{tabular}{|c|c|c|c|c|}
\hline Variable & $\begin{array}{l}\text { Valores iníciales } \\
\text { Media } \pm \text { DE }\end{array}$ & $\begin{array}{l}\text { Valores después de la suplementación } \\
\text { Media } \pm \text { DE }\end{array}$ & IC $95 \%$ & p \\
\hline Peso seco & $29.148 \pm 12.84 \mathrm{~kg}$ & $29.992 \pm 13.04 \mathrm{~kg}$ & $-0.844 .(-1.397-0.290)$ & $0.004^{*}$ \\
\hline Talla & $1.27 \pm .17 \mathrm{~m}$ & $1.28 \pm .18 \mathrm{~m}$ & $-0.006(-0.014-0.001)$ & 0.088 \\
\hline $\mathrm{CB}$ & $19.24 \pm 4.51 \mathrm{~cm}$ & $19.22 \pm 4.59 \mathrm{~cm}$ & $0.020(-0.268 / 0.308)$ & 0.088 \\
\hline PCT & $6.58 \pm 4.11 \mathrm{~mL}$ & $7.04 \pm 4.00 \mathrm{~mL}$ & $-0.460(-1.084 / 0.164)$ & 0.142 \\
\hline IMC & $16.89 \pm 3.85 \mathrm{~kg} / \mathrm{m}^{2}$ & $17.43 \pm 3.96 \mathrm{~kg} / \mathrm{m}^{2}$ & $-0.545(-1.06 /-0.026)$ & $0.040^{*}$ \\
\hline $\begin{array}{l}\text { Bajo peso } \mathrm{n}(\%) \\
\text { Adecuado } \mathrm{n}(\%)\end{array}$ & $\begin{array}{c}16(64 \%) \\
9(36 \%)\end{array}$ & $\begin{array}{r}16(64 \%) \\
9(36 \%)\end{array}$ & & $\begin{array}{l}N S^{* *} \\
N S^{* *}\end{array}$ \\
\hline $\mathrm{AMB}$ & $2401.60 \pm 1033.59 \mathrm{~mm}^{2}$ & $2391.47 \pm 1049.94 \mathrm{~mm}^{2}$ & $\begin{array}{c}10.13 \\
(-102.11 / 122.38)\end{array}$ & 0.854 \\
\hline $\mathrm{AAB}$ & $675.57 \pm 530.29 \mathrm{~mm}^{2}$ & $685.52 \pm 506.95 \mathrm{~mm}^{2}$ & $\begin{array}{c}-9.95 \\
(-65.20 / 45.28)\end{array}$ & 0.713 \\
\hline
\end{tabular}

*T de student: nivel de significancia; ** Cálculo por proporciones. CB: pliegue cutáneo tricipital; IMC: índice de masa corporal; AMB: área muscular de brazo; AAB: área adiposa de brazo.

Cuadro 3. Comparación en los indicadores bioquímicos pre y post de la aplicación con L-carnitina intravenosa en los participantes $(n=25)$

\begin{tabular}{|c|c|c|c|c|}
\hline Variable & $\begin{array}{l}\text { Valores iníciales } \\
\text { Media } \pm \text { DE }\end{array}$ & $\begin{array}{c}\text { Valores después de la suplementación } \\
\text { Media } \pm \text { DE }\end{array}$ & IC $95 \%$ & $\mathbf{p}^{*}$ \\
\hline $\mathrm{HB}$ & $8.40 \pm 1.55 \mathrm{~g} / \mathrm{L}$ & $8.78 \pm 1.33 \mathrm{~g} / \mathrm{L}$ & $\begin{array}{c}-0.38 \\
(-0.868 / 0.108)\end{array}$ & 0.12 \\
\hline HTO & $4.00 \pm .59 \mathrm{~L} / \mathrm{L}$ & $4.23 \pm .40 \mathrm{~L} / \mathrm{L}$ & $\begin{array}{c}-0.23 \\
(-0.44 /-0.03)\end{array}$ & 0.91 \\
\hline Albúmina & $26.56 \pm 4.73 \mathrm{mg} / \mathrm{L}$ & $26.47 \pm 5.01 \mathrm{mg} / \mathrm{L}$ & $\begin{array}{c}0.09 \\
(-1.65 / 1.84)\end{array}$ & $0.02^{* *}$ \\
\hline TGC & $151.28 \pm 44.95 \mu \mathrm{mol} / \mathrm{L}$ & $170.76 \pm 42.58 \mu \mathrm{mol} / \mathrm{L}$ & $\begin{array}{c}-19.48 \\
(-41.35 / 2.39)\end{array}$ & 0.07 \\
\hline Colesterol & $148.24 \pm 41.94 \mu \mathrm{mol} / \mathrm{L}$ & $132.48 \pm 38.47 \mu \mathrm{mol} / \mathrm{L}$ & $\begin{array}{c}15.76 \\
(6.10 / 25.4)\end{array}$ & $0.003^{* *}$ \\
\hline $\mathrm{Na}$ & $140.56( \pm 4.60) \mathrm{mEq} / \mathrm{L}$ & $136.23 \pm 27.47 \mathrm{mEq} / \mathrm{L}$ & $\begin{array}{c}4.32 \\
(-7.3 / 16.01)\end{array}$ & 0.45 \\
\hline K & $4.86 \pm 1.12 \mathrm{mEq} / \mathrm{L}$ & $6.48 \pm 5.58 \mathrm{mEq} / \mathrm{L}$ & $\begin{array}{c}-1.62 \\
(-4.03 / 0.79)\end{array}$ & 0.17 \\
\hline CA & $8.98 \pm 1.28 \mathrm{mg} / \mathrm{dL}$ & $8.86 \pm 1.22 \mathrm{mg} / \mathrm{dL}$ & $\begin{array}{c}0.120 \\
(-0.27 / 0.51)\end{array}$ & 0.53 \\
\hline$P$ & $5.44 \pm 1.89 \mathrm{mg} / \mathrm{dL}$ & $6.21 \pm 2.01 \mathrm{mg} / \mathrm{dL}$ & $\begin{array}{c}-0.76 \\
(-1.52 /-0.006)\end{array}$ & $0.04^{*}$ \\
\hline Urea & $121.21 \pm 40.44 \mathrm{mg} / \mathrm{dL}$ & $133.99 \pm 35.89 \mathrm{mg} / \mathrm{dL}$ & $\begin{array}{c}-12.78 \\
(-26.59 / 0.83)\end{array}$ & 0.06 \\
\hline Creatinina & $7.81 \pm 2.43 \mathrm{mg} / \mathrm{dL}$ & $8.96 \pm 2.63 \mathrm{mg} / \mathrm{dL}$ & $\begin{array}{c}-1.15 \\
(-2.18 /-0.13)\end{array}$ & $0.02 *$ \\
\hline
\end{tabular}

*T de student= nivel de significancia**, $\mathrm{HB}=$ hemoglobina, $\mathrm{HTO}=$ hematocrito, $\mathrm{TGC}=$ triglicéridos, $\mathrm{Na}=$ sodio, $\mathrm{K}=$ potasio, $\mathrm{Ca}=$ calcio, $\mathrm{P}=$ potasio. 
Cuadro 4. Comparación de la eritropoyetina y el paquete globular, pre y post la suplementación con L-carnitina

\begin{tabular}{l|c|c|c|c} 
& Pre & Post & & \\
\hline & \multicolumn{2}{|c|}{ Intervención } & & \\
\hline Variable & Mediana & Mediana & Valor Z & Valor $\mathbf{p}^{*}$ \\
\hline EPO & $2640 \pm 1113$ & $2560 \pm 1083$ & -1 & 0.317 \\
PG & $0.64 \pm 0.86$ & $0.16 \pm 0.55$ & -2.92 & $0.003^{*}$
\end{tabular}

*Con prueba de rangos de Wilcoxon = valor de significancia; EPO: eritropoyetina; PG: paquete globular.

Cuadro 5. Resultados de la correlación entre variables antropométricas con variables bioquímicas

\begin{tabular}{|l|c|c|c|}
\hline Variable 1 & Variable 2 & $\begin{array}{c}\text { Coeficiente } \\
\text { de Pearson }\end{array}$ & P* \\
\hline Peso seco inicial & Creatinina inicial & -0.43 & 0.01 \\
& Urea final & -0.43 & 0.01 \\
& Creatinina final & 0.036 & 0.03 \\
\hline Talla inicial & P inicial & -0.45 & 0.01 \\
& Creatinina inicial & 0.42 & 0.01 \\
& Colesterol final & -0.5 & 0.005 \\
AMB inicial & Creatinina final & 0.43 & 0.01 \\
& Na inicial & -0.5 & 0.005 \\
& Creatinina inicial & 0.37 & 0.03 \\
HB inicial & Urea final & -0.40 & 0.02 \\
\hline TGC inicial & Albumina inicial & 0.45 & 0.01 \\
& Creatinina inicial & -0.44 & 0.01 \\
Colesterol inicial & Creatinina final & -0.41 & 0.01 \\
\hline Talla final & Urea final & 0.47 & 0.008 \\
& Creatinina final & 0.48 & 0.007
\end{tabular}

${ }^{*} \mathrm{p}=$ análisis por correlación de Pearson.

CB: pliegue cutáneo tricipital; IMC: índice de masa corporal; AMB: área muscular de brazo; AAB: área adiposa de brazo; HB: hemoglobina; HTO: hematocrito; TGC: triglicéridos; Na: sodio; K: potasio; Ca: calcio; P: potasio; PG: paquete globular.

\section{Aportaciones complementarias}

Aunque no fue el objetivo principal, los familiares manifestaron una mejora en los síntomas relacionados con la insuficiencia renal crónica, por ejemplo, que mejoró la adinamia, el estado anímico, la coloración cutánea. Respecto a los eventos adversos, náusea y vómito, sólo se pre-
Cuadro 6. Comparación de los principales indicadores que mejoraron a los tres meses de la suplementación con Lcarnitina y la reducción del riesgo atribuible $(\mathrm{N}=25)$

\begin{tabular}{|c|c|c|c|c|}
\hline & $\begin{array}{l}\text { Inicial } \\
\mathbf{N}(\%)\end{array}$ & $\begin{array}{c}\text { A los } 3 \text { meses } \\
\text { de la suple- } \\
\text { mentación } \\
\text { N (\%) }\end{array}$ & RRA & P¥ \\
\hline $\begin{array}{l}\text { Peso seco } \\
\text { Adecuado }\end{array}$ & $9(36)$ & $19(76) *$ & $40 \%$ & 0.010 \\
\hline $\begin{array}{l}\text { IMC } \\
\text { Adecuado }\end{array}$ & $9(36)$ & $15(60)$ & $24 \%$ & NS \\
\hline $\begin{array}{l}\text { Valores de } \\
\text { hemoglobina } \\
\text { Adecuado }\end{array}$ & 0 & $10(40)$ & $60 \%$ & 0.034 \\
\hline Albumina baja & $5(20)$ & $2(8)$ & $12 \%$ & NS \\
\hline $\begin{array}{l}\text { Triglicéridos } \\
\text { Valores por } \\
\text { arriba de los } \\
\text { recomendado }\end{array}$ & $4(16)$ & $17(68)$ & $52 \%$ & 0.0001 \\
\hline Colesterol*** & $\begin{array}{c}2(8) \\
\text { (por arriba } \\
\text { de las } \\
\text { recomen- } \\
\text { daciones) }\end{array}$ & $\begin{array}{c}19 \text { (76) } \\
\text { (disminución } \\
\text { de los valores) }\end{array}$ & $68 \%$ & 0.0001 \\
\hline
\end{tabular}

p¥: comparación por proporciones con el paquete estadístico ${ }^{\circledR}$ PRIMER McGrawHill Interamericana (1998), entre los valores iniciales y a los tres meses de la suplementación. RRA: la reducción del riesgo atribuible.

*Aumento o mejora considerada cuando se presentó un incremento igual o mayor a $0.5 \mathrm{~kg}$.

**Mejoría considerada cuando hubo un incremento en los valores de hemoglobina a los tres meses de la suplementación $\geq$ a $1 \mathrm{~g} / \mathrm{dL}$.

***Aunque con la intervención siguieron con cifras por arriba de lo recomendado, en un $76 \%$ hubo disminución de las cifras en un rango de $12-54 \mathrm{mg} / \mathrm{dL}$.

sentó un caso, registrando que, para este caso, la L-carnitina se aplicó en bolo.

\section{DISCUSIÓN}

El deterioro nutricional en los niños con insuficiencia renal crónica es prevalente y tiene un mayor impacto de acuerdo al grupo de edad y las demandas energéticas que conlleva de acuerdo a la edad de cada paciente, por lo que las alternativas para mejorar el estado nutricio se vuelven imprescindibles. ${ }^{5}$ 
Acorde a los resultados observados, los valores de hemoglobina no presentaron una mejoría a diferencia de los estudios por Aoun y sus colegas, ${ }^{13}$ lo cual se puede explicar por el tamaño de muestra y por la duración de la suplementación que fue de 6 meses. Al realizar la comparación por proporciones y la medición de la reducción en el riesgo hubo una significancia clínica para la hemoglobina, lo cual es importante considerar, para ajustar los tiempos y un tamaño de muestra adecuado con estos hallazgos.

Una fortaleza de nuestro estudio comparado con el de Aoun y sus colaboradores es que se consideró desde un inicio que los participantes no estuvieran recibiendo tratamiento con hierro, lo cual pudo explicar la mejora observada en estos autores. Por otro lado, aunque una limitante fue el no contar con un tamaño de muestra adecuado, vale la pena mencionar que ésta en nuestro estudio sigue siendo mayor a la observada en la mayoría de los estudios (menor a 15 participantes) y donde tampoco hacen mención a un cálculo de tamaño de la muestra.

Por otro lado, Lilien y su equipo ${ }^{14}$ tampoco encontraron una diferencia significativa en los niveles de hemoglobina ni una reducción en la dosis de eritropoyetina, lo cual coincide en este estudio; sin embargo, es pertinente mencionar que se pensó que se podrían obtener resultados favorables, ya que la vía de suplementación de la L-carnitina en el estudio de Lilien y su grupo ${ }^{14}$ fue vía oral, y en nuestro estudio fue vía intravenosa, cuya farmacocinética es mejor acorde a la biodisponibilidad y estabilidad en la absorción, ${ }^{15}$ lo cual se pudo comprobar al hacer el cálculo por proporciones para los casos y se pudieron detectar mejorías para hemoglobina después de la maniobra con incremento de $0.1 \mathrm{a} 2.9 \mathrm{~g} / \mathrm{dL}$, lo cual refleja un beneficio con la suplementación.

A pesar de la evidencia científica, de la falta de efectos de la suplementación con L-carnitina sobre el estado nutricio de los pacientes, puede explicarse porque se consideran sólo los parámetros de peso para la talla, talla para la edad y peso para la edad, ${ }^{16}$ y nuestro estudio analizó un conjunto de variables que se correlacionaron con el estado nutricio, encontrando diferencia significativa para el peso seco, índice de masa corporal y niveles de albúmina, creatinina y colesterol. Las variables en conjunto permiten observar un probable efecto benéfico sobre la mejoría al estado nutricio, con la suplementación de L-carnitina con un corto tiempo de intervención (3 meses). El resto de las variables antropométricas como el área muscular de brazo y el área adiposa del brazo no mostraron una mejora significativa, pero se sugiere seguir estudiando en el seguimiento a largo plazo, y un indicador de efectos a corto plazo, pudieran ser a través de los niveles de creatinina, que para este estudio tuvieron un incremento, sobre todo al hacer a correlación, posterior a la intervención con el peso, talla e índice de masa corporal, lo cual pudiera ser un marcador indirecto del aumento de la masa muscular. ${ }^{16}$

Para los valores de colesterol y triglicéridos, nuestros hallazgos no concuerdan con lo reportado por Gunes y sus colegas en el 2005. ${ }^{17}$ Para los triglicéridos no se encontró una reducción significativa en sus valores al final de la maniobra, a diferencia de los valores de colesterol que sí se observó una reducción significancia; esto se puede deber a que los pacientes con insuficiencia renal crónica presentan una deficiencia de carnitina y al ser hemodializados, ésta traspasa el filtro, lo cual explica esta falta de reducción en los niveles de triglicéridos, y al no haber una deficiencia de la carnitina, la función de facilitar la entrada a los triglicéridos de cadena media a la matriz mitocondrial y que comience el proceso de beta oxidación, ${ }^{5}$ se encuentra alterada, por lo que al ser suplementada se evitan estas dificultades facilitando el proceso y reduciendo los 
niveles de colesterol. Al hacer la comparación y análisis por la cantidad (porcentaje) de casos que mostraron disminución después de la maniobra, se observaron reducciones de 15 a $81 \mathrm{mg} / \mathrm{dL}$ en siete casos para los triglicéridos y, de 15 casos $(60 \%)$ para el colesterol de 6 a $54 \mathrm{mg} / \mathrm{dL}$, lo cual es un hallazgo clínico.

Otros de los bioquímicos fue el fosforo, el cual presentó un incremento en sus cifras, una razón se explica a que dicho elemento presenta variabilidad en su ingestión dietética y una limitante del estudio, fue el no contar con un registro del consumo dietético de este elemento y otros nutrimentos, por lo que sugerimos que este hallazgo se pudo deber a la ingestión de alimentos ricos en fosforo un día previo a la sesión de hemodiálisis. Por lo que esto se podría controlar en otros diseños al registrar y analizar el consumo real de alimentos en los participantes. ${ }^{17}$

Los indicadores que se esperaba contar con una diferencia significativa no se lograron con la estadística utilizada (t de student) por el pequeño tamaño de los casos de estudio, por lo que al mejorar la comparación por proporciones de los diferentes marcadores bioquímicos y antropométricos antes y después de la maniobra, se pudo detectar que hubo mejoría en la mayoría de los casos de estudio para la hemoglobina, albúmina, peso seco, colesterol y triglicéridos, con una disminución de reducción en el riesgo del 12 al 68\% de los casos de estudio.

Los estudios sobre la seguridad a la administración L-carnitina, indican que los efectos adversos son bajos, para este estudio solo se presentó un evento adverso relacionado con la L-carnitina y fue la presencia de náusea y vómito. Este evento adverso no serio, que la literatura ha registrado, se debe a la técnica de aplicación de la Lcarnitina, en la que se suele usar bolo en lugar de la infusión. ${ }^{11}$
Finalmente, aunque no fue uno de los objetivos principales, los familiares mencionaron una mejora en los síntomas relacionados con la insuficiencia renal crónica, por ejemplo que mejoró la adinamia, el estado anímico, la coloración cutánea y diminución en la anorexia. Por lo que sería interesante que se puedan medir a largo plazo la mejora en la calidad de vida, al generar un impacto al estado nutricio y otros marcadores metabólicos como el equilibrio en lípidos, la glucosa y la anemia.

\section{CONCLUSIONES}

La suplementación con L-carnitina intravenosa de $50 \mathrm{mg} / \mathrm{kg} / \mathrm{sesión,} 30$ minutos antes de terminar cada sesión de hemodiálisis, durante tres meses, permitió una mejora en el porcentaje de los casos para peso seco, índice de masa corporal, albúmina, hemoglobina y una reducción para los casos de colesterol y triglicéridos; con una reducción del riesgo atribuible del 12 al 68\% de los casos (pre y post maniobra).

\section{Agradecimientos}

Al Dr. Alejandro Serra Martínez, quien en su momento era Jefe de Hemodiálisis de la Unidad Médica de Alta Especialidad de Gineco-Pediatría \# 48 quien otorgó todas las facilidades para realizar el estudio.

A todo el personal que labora en el área de hemodiálisis de la misma unidad médica (enfermeras, médicos, trabajadoras sociales, asistentes médicas).

\section{REFERENCIAS}

1. Troche A, Ávalos D, Ferreira S, Zarza de Bolaños M .Características epidemiológicas de la insuficiencia renal crónica (IRC) en Pediatría. Rev Parag Pediatr. 2005(32):122-39.

2. Martínez GT, García JA. Desnutrición energético-proteínica. En: Casanueva E, Kaufere M, Pérez AB, Arroyo P. Nutriología Médica. 3a edición. México: Médica Panamericana; 2008. p. 263-295. 
3. Susan M, Geary DF. Anemia in children with chronic kidney disease. Pediatr Nephrol 2008;23(2):209- 219.

4. Caló LA, Vertolli U, Davis PA, Savica V. L carnitine in hemodialysis patients. Hemodial Int. 2012;16(3):428-34.

5. Fischbach M, Edefonti A, Schroder C, Watson A and The European Pediatric Dialysis Working Group. Hemodialysis in children: general practical guidelines. Pediatr Nephrol. 2005;20(8):1054-1066.

6. National Center for Toxicological Research. Annual. Report. http://www.fda.gov/downloads/AboutFDA/CentersOffices/OC/OfficeofScientificandMedicalPrograms/NCTR/ ResearchAccomplishmentsPlans/UCM257438.pdf

7. COFEPRIS. Relación de certificados de libre venta emitidos durante el 2do semestre del 2011. http://www.encuentra. gob.mx/resultsAPF.html?q=levocarnitina\&client=cofepris

8. Hurot JM, Cucherat M, Haugh M, Fouque D. Effects of L-Carnitine Supplementation in Maintenance Hemodialysis Patients: A Systematic Review. J Am Soc Nephrol.2002;13(3):708-714.

9. Matsura M, Hatakeyama S, koni I, Mabuchi H, Muramoto $\mathrm{H}$. Correlation between serum carnitine levels and erythrocyte osmotic fragility in hemodialysis Patients. J Nephrol. 2008;72(4):5.

10. Kathleen $L$ and EscottS. Terapia nutricional médica en trastornos renales. In: Wilkens $\mathrm{K}$ and Juneja V. (eds.) Krause Dietoterapia. 12th ed. Barcelona, España: MASSON; 2009:921-959.
11. Sabry AA. The role of oral L-Carnitine therapy in chronic hemodialysis patients. Saudi J kidney Dis transpl.2010;21(3):454-459.

12. Ávila H, Caraveo VE, Valcez R, Tejero E. Evaluación del estado de nutrición. En: Casanueva E, Kaufere M, Pérez AB, Arroyo P. Nutriología Médica. 3ạ edición. México: Médica Panamericana; 2008. p. 747-783.

13. Aoun B, Berard E, Vitkevic R, Dehée A, Bensman A, Ulinski $T$. L-carnitine supplementation and EPO requirement in children on chronic hemodialysis. Pediatr Nephrol 2010;25:557-560.

14. Lilien MR, Duran M, Quak JM, Frankhuisen JJ, Schröder CH. Oral L-carnitine does not decrease erythropoietin requirement in pediatric dialysis. Pediatr Nephrol 2000;15:17-20.

15. Nikolaos S, George A, Telemachos T, Maria S, Yannis M, Konstantinos M. Effect of L-carnitine supplementation on red blood cell deformability in hemodialysis patients. Ren Fail 2000;22(1):73-80.

16. Blumenkrantz MJ, Kopple JD, Gutman RA, Chan YK, Barbour GL, Roberts $\mathrm{C}$ y cols. Methods for assessing nutritional status of patients with renal failure. Am J Clin Nutr 1980;33(7):1567-1585.

17. Günes B, Yalcin SS, kalkanoglu HS, Onol S, Dursun A, Coşkun $T$. The effect of oral L-carnitine supplementation on the lipid profiles of hyperlipidaemic in children. Acta Paediatrica 2005;94(6):711-716. 